Reprod. Nutr. Dévelop., 1987, 27 (1 B), 233-234.

\title{
Influence du monensine sur le transit de la phase liquide du rumen
}

Michaela ROGERS, J.-P. JOUANY

Laboratoire de la Digestion des Ruminants, I.N.R.A. Theix 63122 Ceyrat, France.

Summary. Monensin had no effect on either the distribution volume of polyethyleneglycol (PEG) or the duodenal flow of liquid. Monensin supplementation increased the mean retention time of PEG in the rumen but this effect disappeared after the ionophore was withdrawn.

Le monensine a fait l'objet de nombreux travaux au cours de ces dernières années mais son mode d'action chez le ruminant n'est pas encore totalement expliqué (Schelling, 1984). Leménager et al. (1978) ont montré que l'addition de cet antibiotique modifie le temps de séjour des aliments dans le rumen, ce qui pourrait expliquer certains changements dans le métabolisme du rumen et les quantités d'aliments ingérées. En outre, la plupart des études ont été effectuées sur de courtes périodes alors que le monensine est distribué dans la pratique pendant plusieurs mois. C'est pourquoi nous avons entrepris d'étudier in vivo l'action du monensine sur le transit de la phase liquide du rumen en fonction de la durée de sa distribution, et de vérifier la possibilité d'un effet rémanent après arrêt de sa distribution.

Matériel et méthodes. Deux régimes (témoin et expérimental) ont été distribués en deux repas $\left(45 \mathrm{~g} / \mathrm{jour} / \mathrm{kg} \mathrm{P}^{0,75}\right)$ à 2 lots de 4 moutons Texel, adultes, qui portaient des canules du rumen et du duodénum. Les régimes, présentés sous forme agglomérée, contenaient du foin de prairie naturelle $(44 \%)$, du maïs grain $(35 \%)$ et des graines de lupin $(21 \%)$. Le taux d'incorporation du monensine dans le régime expérimental était de $36 \mathrm{ppm}$.

Les animaux du lot 1 (témoin) ont été nourris avec l'aliment témoin pendant toute la durée de l'expérience qui a été répartie en 4 périodes. Au cours de la période 1 (préexpérimentale), l'ensemble des animaux du lot 1 et du lot II ont reçu I'aliment témoin. Les animaux du lot II ont été alimentés avec l'aliment expérimental pendant les périodes 2 et 3 correspondant respectivement à des durées de distribution de monensine de 19 à 96 jours, puis ils ont reçu le régime témoin pendant la période 4 (postexpérimentale).

L'infusion de polyéthylèneglycol (PEG) $(24 \mathrm{~g} / \mathrm{j})$ a débuté 5 jours avant la mesure du taux de récupération dans les fèces. Les prélèvements de contenus duodénaux ont été réalisés pendant 2 jours toutes les $4 \mathrm{~h}$ de façon à constituer un échantillon moyen par animal. Les cinétiques de décroissance de la concentration du marqueur dans les contenus duodénaux ont été effectuées aux temps $0,2,4$, $6,12,24$ et $30 \mathrm{~h}$ après arrêt de l'infusion. Le PEG a été dosé selon la méthode de Hyden (1955) et les calculs ont été faits selon les recommandations de Faichney (1975). Les effets " monensine ", " durée de la distribution " et leurs interactions ont été mesurés par analyse de variance.

Résultats et discussion. II n'y a pas eu d'effet « monensine " ou d'interaction " monensine $x$ durée de distribution " sur le volume du rumen, ni sur le flux de la 
phase liquide. L'existence d'une interaction significative $(P<0,01)$ " antibiotique $x$ durée " sur le temps de rétention moyen de la phase liquide du rumen (TRM) montre que l'effet " monensine » sur ce paramètre évolue avec le temps.

L'augmentation significative du TRM, due au monensine, a été de 40 à $63 \%$ au cours des périodes 2 et 3 . Elle confirme les résultats de Leménager et al. (1978). L'absence d'effet de rémanence sur le TRM après suppression du monensine à la période 4 prouve qu'il est nécessaire de le distribuer en continu pour que son action se maintienne. Au sein du lot II, la différence entre la période 2 et 3 qui reflète l'effet "durée " de distribution du monensine sur le TRM, n'a pas été significative. Ce résultat traduit l'absence d'adaptation du rumen au monensine distribué pendant 96 jours, mais les changements importants du TRM observés sur l'ensemble des animaux au cours de la période 3, probablement dus à une diminution de la témpérature ambiante, ne permettent pas de tirer des conclusions précises sur l'effet d'une distribution à long terme de l'antibiotique.

L'examen des valeurs du volume du rumen et du flux de la phase liquide (tabl. 1) permet de penser que l'antibiotique peut avoir à la fois une action sur le volume du rumen (augmentation) et sur le flux de liquide (diminution). Le seuil de signification de ces 2 paramètres n'aurait pas été atteint à cause du nombre limité de données et des variations individuelles entre animaux.

TAB. 1. - Effet du monensine sur le volume et le renouvellement de la phase liquide du rumen.

\begin{tabular}{|c|c|c|c|c|c|}
\hline & Animaux & Période 1 & Période 2 & Période 3 & Période 4 \\
\hline Volume (I) & $\begin{array}{l}\text { témoins } \\
\text { expérimentaux }\end{array}$ & $\begin{array}{l}8,6 \pm 1,1 \\
9,6 \pm 1,2\end{array}$ & $\begin{array}{l}10,1 \pm 3,7 \\
11,0 \pm 1,8^{*}\end{array}$ & $\begin{array}{c}6,3 \pm 1,2 \\
10,2 \pm 1,0^{* *}\end{array}$ & $\begin{array}{l}7,3 \pm 0,8 \\
8,1 \pm 1,2\end{array}$ \\
\hline Flux $(\mathrm{kg} / \mathrm{j})$ & $\begin{array}{l}\text { témoins } \\
\text { expérimentaux }\end{array}$ & $\begin{array}{l}15,1 \pm 2,1 \\
14,0 \pm 1,0\end{array}$ & $\begin{array}{l}15,5 \pm 2,5 \\
12,8 \pm 1,1^{*}\end{array}$ & $\begin{array}{l}13,9 \pm 1,6 \\
13,0 \pm 1,4^{* *}\end{array}$ & $\begin{array}{l}13,1 \pm 1,1 \\
12,7 \pm 1,1\end{array}$ \\
\hline $\operatorname{TRM}(\mathrm{h})$ & $\begin{array}{l}\text { témoins } \\
\text { expérimentaux }\end{array}$ & $\begin{array}{l}14,2 \pm 1,0 \\
16,7 \pm 1,4\end{array}$ & $\begin{array}{l}14,8 \pm 2,3 \\
20,8 \pm 2,2^{*}\end{array}$ & $\begin{array}{l}11,2 \pm 1,6 \\
18,2 \pm 1,2^{*}\end{array}$ & $\begin{array}{l}14,0 \pm 1,1 \\
15,5 \pm 1,3\end{array}$ \\
\hline
\end{tabular}

Durée de la distribution de monensine : * $: 19$ jours ; ** $: 96$ jours.

L'augmentation nette du TRM observée chez les animaux ayant reçu le monensine peut expliquer l'évolution des paramètres digestifs dans le rumen au même titre que l'action " antibiotique " bien connue de cette molécule. Les deux effets sont parfois additifs (diminution de l'efficacité de la synthèse microbienr,e, modification de l'équilibre microbien) ou s'opposent (dégradation des aliments). Ces actions synergiques ou antagonistes peuvent être à l'origine de résultats parfois divergeants entre auteurs, concernant l'action du monensine en fonction de la nature des rations.

Hyden S., 1955. Kgl. lantbrukes. Hogskol. Ann., 22, 139.

Faichney G. J., 1975. In Mc Donald and Warner (ed.). Digestion and metabolism in the ruminant. Univ. New England, Armidale.

Leménager R. P., Owens F. N., Shockey B. J., Lusby K. S., Totusek R., 1978. J. anim. Sci., 47, 255.

Schelling G. T., 1984. J. anim. Sci, 58, 1518. 